\title{
Tonsillar Tuberculosis Associated with Pulmonary and Laryngeal Foci
}

\author{
Koji Yamamoto, Fumihiro Iwata, Atsushi Nakamura*, Yasuhito Iwashima, Tomokatsu Mryaki, \\ Hisashi Yamada, Miyuki Kurachi, Yasumasa Sato, Katsuhiko Tsukada, Toshihiko Takeuchi, \\ Takashi JoH*, Yoshifumi YoкоYамA* and Makoto Iтон*
}

\begin{abstract}
Tonsillar tuberculosis is one of the uncommon forms of extrapulmonary tuberculosis. We report a case of tonsillar tuberculosis associated with pulmonary and laryngeal foci. A 23-year-old female was admitted for evaluation of hoarseness and difficulty in swallowing. Bilateral palatine tonsils were enlarged, and a tonsillectomy was performed. Since a histological study revealed tonsillar tuberculosis, antituberculous agents were administered. After the treatment the pulmonary lesions detected with chest computed tomography were improved, and her symptoms were relieved. The possibility of tonsillar tuberculosis should be considered when unexplained enlarged tonsil is observed in patients with pulmonary tuberculosis.

(Internal Medicine 41: 664-666, 2002)
\end{abstract}

Key words: chest computed tomography, laryngoscopy, acidfast bacilli, antituberculous agents

\section{Introduction}

Tuberculosis has been one of the most serious infectious diseases in the world (1). The World Health Organization declared a state of emergency with regard to tuberculosis in 1993; in Japan alone in a single year (1997), approximately 42,000 new cases were reported. Tonsillar tuberculosis is one of the uncommon forms of extrapulmonary tuberculosis; a few cases of tonsillar tuberculosis have been reported recently in Japan.

In this report, we describe a rare case of tonsillar tuberculosis associated with pulmonary and laryngeal foci. A detailed review of the literature on tonsillar tuberculosis is also included.

For editorial comment, see p 606.

\section{Case Report}

A previously well 23-year-old woman was admitted on May 24, 1999 to the Department of Internal Medicine at NTT West Tokai Hospital for evaluation of hoarseness and difficulty in swallowing of two months' duration.

There were no relevant family or past medical histories. The patient never smoked and seldom drank alcoholic beverages. On admission, her height and weight were $160 \mathrm{~cm}$ and $49 \mathrm{~kg}$, respectively, her body temperature was $36.5^{\circ} \mathrm{C}$, her pulse was 70 beats/min, and her blood pressure was $102 / 64 \mathrm{mmHg}$. The head, lungs, heart, and abdomen appeared grossly normal.

Laboratory findings on admission were as follows: hematocrit $37.6 \%$; white blood cell count $3,750 / \mathrm{mm}^{3}$; platelet count $24.4 \times 10^{4} / \mathrm{mm}^{3}$; serum total protein $7.7 \mathrm{~g} / \mathrm{dl}$; serum aspartate aminotransferase $15 \mathrm{IU} / l$; serum alanine aminotransferase 10 IU/l; lactic dehydrogenase $240 \mathrm{IU} / l$; total bilirubin $0.5 \mathrm{mg} / \mathrm{dl}$; serum alkaline phosphatase $151 \mathrm{IU} / \mathrm{l}$; C-reactive protein 0.1 $\mathrm{mg} / \mathrm{dl}$; and erythrocyte sedimentation rate $9 \mathrm{~mm}$ in the 1 st hour.

Purified protein derivative skin test was negative, and smears and cultures of sputum were also negative for acid-fast bacilli, including the testing with polymerase chain reaction (PCR). Human immunodeficiency virus (HIV) tested by enzyme linked immunosorbent assay (ELISA) was negative. Oral examination revealed the enlargement of bilateral palatine tonsils, and laryngoscopy revealed the presence of edema in the epiglottis. For the relief of her symptoms and the histological diagnosis, a tonsillectomy under general anesthesia was performed on June 6,1999 . Histological examination of the resected specimen showed granuloma formation with epithelioid cells and Langhans' giant cells, lymphocytic infiltration and caseous necrosis (Fig. 1). Although the testing with PCR for tuberculosis was negative, acid-fast bacilli were detected by ZiehlNeelsen staining on the tissue samples. These histological findings confirmed tonsillar tuberculosis, and there was no evidence of malignancy.

From the Department of Internal Medicine, NTT West Tokai Hospital and *the First Department of Internal Medicine, Nagoya City University School of Medicine, Nagoya

Received for publication June 14, 2001; Accepted for publication March 20, 2002

Reprint requests should be addressed to Dr. Koji Yamamoto, the Department of Internal Medicine, NTT West Tokai Hospital, 2-17-5 Matsubara, Naka-ku, Nagoya 460-0017 


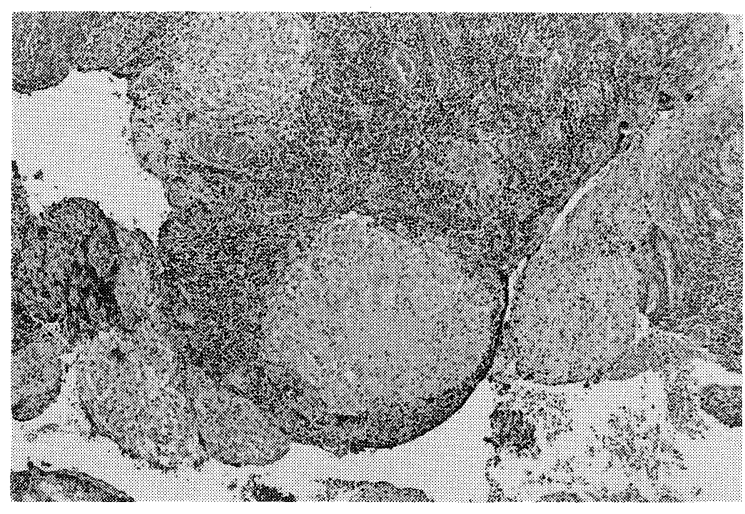

Figure 1. Histological findings of the resected tonsils (HE stain, $\times 100)$. Granuloma formation with epithelioid cells and Langhans' giant cells, lymphocytic infiltration and caseous necrosis were observed.

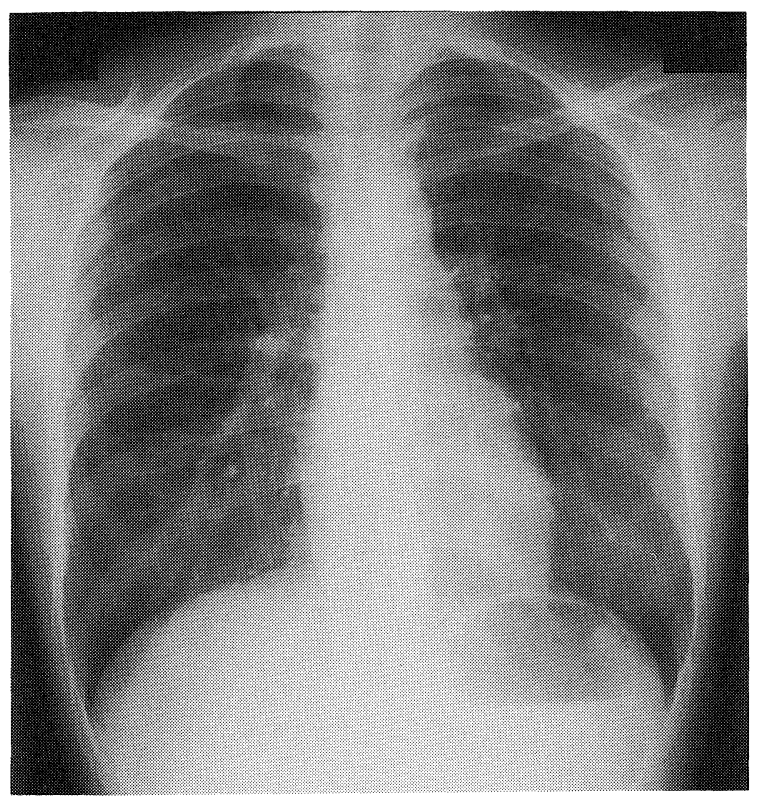

Figure 2. Chest X-ray showed no abnormalities.

Although chest X-ray showed no abnormalities (Fig. 2), chest computed tomography (CT) showed infiltrative shadows in the middle lobe of the right lung, and in the lower lobe (S6, S9, S10) of both lungs, which were compatible with pulmonary involvement of tuberculosis (Fig. 3). Based on these findings of histological examination, chest CT, and laryngoscopy, a diagnosis of tonsillar tuberculosis associated with pulmonary and laryngeal lesions was made. Administration of antituberculous agents (isoniazid $400 \mathrm{mg} / \mathrm{day}$, rifampicin $450 \mathrm{mg} / \mathrm{day}$, pyrazinamide $1,500 \mathrm{mg} / \mathrm{day}$, and streptomycin $1,000 \mathrm{mg} /$ day) was given without any side effect. After the initial four weeks

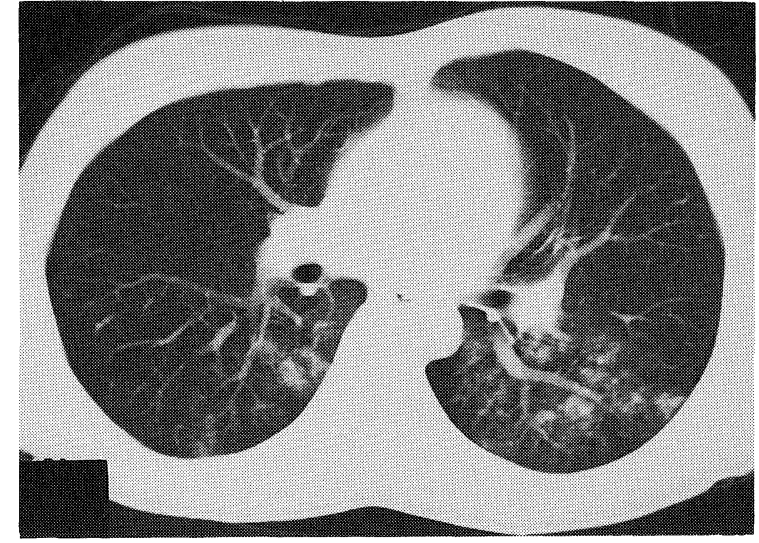

Figure 3. Chest computed tomography showed infiltrative shadows in both lungs.

treatment, chest CT and laryngoscopy revealed the improvement of the infiltrative shadows in the lung field and the edema in the epiglottis, respectively. Also, the symptoms of the patient were relieved after the treatment. The patient has been free of recurrence after the completion of treatment on January 4, 2000.

\section{Discussion}

Although the improvement of lifestyle and medical and pharmaceutical advances has dramatically decreased the number of tuberculosis cases, it has still been one of the major infectious diseases all over the world, including Japan.

Tonsillar tuberculosis is a rare disease recently. Tuberculosis of the oral cavity has shown a decline in several parts of the world, concomitant with the decreasing incidence of pulmonary tuberculosis. This has been attributed to the advent of specific chemotherapy to combat tuberculosis, improved surveillance, early and effective treatment and decreased infectivity (2). Also, the upper respiratory tract is generally resistant to tuberculosis (3). The presence of saliva and saprohytes, the antagonism of the striated musculature, and the thickness of the protective epithelial layer are thought to have an inhibitory effect on tubercle bacilli $(3,4)$.

The recent increase in the cases with HIV infection has led to a resurgence of tuberculosis. Concomitant HIV infections are present in $25 \%$ of patients with laryngeal tuberculosis (5). The present case had no history of HIV infection, and HIV tested by ELISA was negative. The commonest local symptoms of tonsillar tuberculosis are hoarseness and difficulty in swallowing (2), both of which were seen in our case.

Histological examination and the identification of tubercle bacilli usually provide the most conclusive evidence for the diagnosis (2). In the present case, the histological findings of the resected tonsils confirmed the diagnosis of tonsillar tuberculosis. The infiltrative shadows in both lungs detected by chest 
$\mathrm{CT}$ and the edema in the epiglottis shown by laryngoscopy suggested the complication with pulmonary and laryngeal tuberculosis, respectively. Also, the improvement of the findings of chest CT and laryngoscopy after the administration of antituberculous agents confirmed the presence of pulmonary and laryngeal tuberculosis. Since most of tuberculosis of the oral cavity is secondary tuberculosis originating from pulmonary tuberculosis (6), our case is thought to be a case of secondary tonsillar and laryngeal tuberculosis with primary pulmonary tuberculosis. Because of the histological evidence of the tonsils, we would emphasize tonsillar tuberculosis.

In summary, we have presented a rare case of tonsillar tuberculosis associated with pulmonary and laryngeal foci. Tonsillar tuberculosis should be considered as well as malignancy, when enlargement of the palatine tonsils is observed. Chest $\mathrm{CT}$ should be performed, even when chest X-ray shows no abnormalities in patients who have tonsillar tuberculosis.

\section{References}

1) Sutbeyaz Y, Ucuncu H, Murat Karasen R, Gundogdu C. The association of secondary tonsillar and laryngeal tuberculosis: a case report and literature review. Auris Nasus Larynx 27: 371-374, 2000.

2) Rupa V, Bhanu TS. Laryngeal tuberculosis in the eighties-an Indian experience. J Laryngol Otol 103: 864-868, 1989.

3) Verma A, Mann SBS, Radotra B. Primary tuberculosis of the tongue. Ear Nose Throat J 68: 718-720, 1989.

4) Brennan TF, Vrabec DP. Tuberculosis of the oral mucosa. Report of a case. Ann Otol Rhinol Laryngol 79: 601-605, 1970.

5) Singh B, Balwally AN, Nash M, Har-El G, Lucente FE. Laryngeal tuberculosis in HIV-infected patients: a difficult diagnosis. Laryngoscope 106: 1238-1240, 1996.

6) Selimoglu E, Sutbeyaz Y, Ciftcioglu MA, Parlak M, Esrefoglu M, Ozturk A. Primary tonsillar tuberculosis: a case report. J Laryngol Otol 109: 880$882,1995$. 